\title{
OPEN Chronic BDNF simultaneously inhibits and unmasks superficial dorsal horn neuronal activity
}

\begin{abstract}
Sascha R. A. Alles ${ }^{1,2,6 \bowtie}$, Max A. Odem ${ }^{3,6}$, Van B. Lu' ${ }^{2,4}$, Ryan M. Cassidy \& Peter A. Smith ${ }^{2}$
Brain-derived neurotrophic factor (BDNF) is critically involved in the pathophysiology of chronic pain. However, the mechanisms of BDNF action on specific neuronal populations in the spinal superficial dorsal horn (SDH) requires further study. We used chronic BDNF treatment ( $200 \mathrm{ng} / \mathrm{ml}, 5-6$ days) of defined-medium, serum-free spinal organotypic cultures to study intracellular calcium $\left(\left[\mathrm{Ca}^{2+}\right]_{\mathrm{i}}\right)$ fluctuations. A detailed quantitative analysis of these fluctuations using the Frequency-independent biological signal identification (FIBSI) program revealed that BDNF simultaneously depressed activity in some SDH neurons while it unmasked a particular subpopulation of 'silent' neurons causing them to become spontaneously active. Blockade of gap junctions disinhibited a subpopulation of SDH neurons and reduced BDNF-induced synchrony in BDNF-treated cultures. BDNF reduced neuronal excitability assessed by measuring spontaneous excitatory postsynaptic currents. This was similar to the depressive effect of BDNF on the $\left[\mathrm{Ca}^{2+}\right]_{i}$ fluctuations. This study reveals novel regulatory mechanisms of SDH neuronal excitability in response to BDNF.
\end{abstract}

Injury to, or disease of, the somatosensory system frequently generates chronic and sometimes intractable neuropathic pain ${ }^{1,2}$. In experimental animals, peripheral nerve damage, such as that generated by chronic constriction or section of the sciatic nerve, induces pain-related behaviours that serve as a model for human neuropathic pain $^{3,4}$. Seven or more days of sciatic nerve injury promote an enduring increase in the excitability of first order primary afferent neurons ${ }^{5-8}$. These become chronically active and release a variety of mediators (cytokines, chemokines, neuropeptides, ATP and growth factors) that predispose spinal microglia to a more 'activated' state $^{9-14}$. These in turn, release further mediators, including brain derived neurotrophic factor (BDNF) that promote a slowly developing, but persistent increase in excitability of second order neurons in the spinal dorsal horn. This 'central sensitization' is thought to be responsible for the allodynia, hyperalgesia, spontaneous pain and causalgia that characterize neuropathic pain ${ }^{3,15}$. Spinal actions of BDNF involve alteration in $\mathrm{Cl}^{-}$gradients such that the normally inhibitory actions of GABA become excitatory ${ }^{16,17}$. There is also increased excitatory synaptic drive to putative excitatory neurons ${ }^{9,18}$. This results in an overall increase in excitability as monitored by confocal $\mathrm{Ca}^{2+}$ imaging in organotypic cultures of rat or mouse spinal cord ${ }^{9,18-20}$. Despite this, the long-term effects of upregulated BDNF on neuronal plasticity in the superficial dorsal horn (SDH) are not fully understood.

In our previous work, we developed and used a model to study neuropathic pain whereby defined-medium spinal organotypic cultures are treated long-term (5-6 days) with BDNF (200 ng/ml $)^{9,20-23}$. BDNF treatment is administered at day 20 or more after organotypic cultures are established to allow neurons to mature to a more 'adult-like' state. We demonstrated that lamina II neurons in these organotypic cultures are similar to those in acute slices from young adult rats ${ }^{21}$. We also showed that the changes that occur in spinal organotypic dorsal horn neurons 'mirror' those observed in dorsal horn neurons from acute slices obtained from chronic constriction injury (CCI) rats at peak mechanical hypersensitivity levels ${ }^{20}$. Naïve cultures display spontaneous oscillatory changes in intracellular $\mathrm{Ca}^{2+}$ levels $\left[\mathrm{Ca}^{2+}\right]_{\mathrm{i}}$ and we have previously shown that the amplitude and frequency of these changes in $\left[\mathrm{Ca}^{2+}\right]_{\mathrm{i}}$ are profoundly increased following 5-6 days treatment with $\mathrm{BDNF}^{20}$.

We used this model and a new Frequency-independent biological signal identification (FIBSI) ${ }^{24}$ program to quantitatively measure the fluctuations of $\left[\mathrm{Ca}^{2+}\right]_{i}$ and examine their synchronicity. Unexpectedly, we observed

\footnotetext{
${ }^{1}$ Department of Anesthesiology \& Critical Care Medicine, University of New Mexico Health Sciences Center, MSC 10-6000, 2211 Lomas Blvd. NE, ACM 200, Albuquerque, NM 87106, USA. ${ }^{2}$ Neuroscience and Mental Health Institute \& Department of Pharmacology, University of Alberta, Edmonton, AB, Canada. ${ }^{3}$ Department of Microbiology and Molecular Genetics, McGovern Medical School at UTHealth, Houston, TX, USA. ${ }^{4}$ Wellcome-MRC Institute of Metabolic Science, University of Cambridge, Cambridge, UK. ${ }^{5}$ Department of Psychiatry and Behavioral Sciences, Vanderbilt University Medical Center, Nashville, TN, USA. ${ }^{6}$ These authors contributed equally: Sascha R. A. Alles and Max A. Odem. ${ }^{\square}$ email: salles@salud.unm.edu
} 
two opposite effects of BDNF that appeared to occur simultaneously. First, BDNF caused an overall decrease in fluctuation size. Second, we noticed a particular population of SDH neurons in naïve cultures that did not display typical, marked fluctuations of $\left[\mathrm{Ca}^{2+}\right]_{\mathrm{i}}$. This population of 'silent' neurons was never seen in BDNFtreated cultures, suggesting that BDNF unmasks these 'silent' neurons and causes them to become spontaneously active. We next investigated the role of gap junctions in mediating the $\left[\mathrm{Ca}^{2+}\right]_{\mathrm{i}}$ fluctuations; application of the gap junction blocker octanol to chronic BDNF-treated neurons revealed a subpopulation of neurons that generate low-frequency, large $\left[\mathrm{Ca}^{2+}\right]_{\mathrm{i}}$ fluctuations. Further pharmacological experiments indicated the $\left[\mathrm{Ca}^{2+}\right]_{\mathrm{i}}$ fluctuations in active neurons are regulated by diverse mechanisms including voltage-gated calcium and sodium channels as well as GABA and NMDA receptors. Finally, we used FIBSI to reanalyze a previous dataset of spontaneous excitatory postsynaptic current (sEPSC) recordings from BDNF-treated dorsal horn neurons in order to qualitatively compare the effects of BDNF on the sEPSCs and $\left[\mathrm{Ca}^{2+}\right]_{\mathrm{i}}$ fluctuations in SDH neurons. The depressive effects of BDNF on the sEPSCs in delay and tonic-firing SDH neurons were consistent with the effects on the $\left[\mathrm{Ca}^{2+}\right]_{\mathrm{i}}$ fluctuations. This study reveals novel mechanisms of BDNF regulation of dorsal horn excitability, which has implications for the study of chronic neuropathic pain physiology.

\section{Results}

Chronic BDNF treatment of spinal organotypic cultures simultaneously depresses and unmasks $\left[\mathrm{Ca}^{2+}\right]_{i}$ fluctuations in superficial dorsal horn neurons. In previous work we identified that chronic BDNF treatment $\left(200 \mathrm{ng} / \mathrm{ml}, 5-6\right.$ days) induces $\mathrm{Ca}^{2+}$ fluctuations compared to naïve cultures ${ }^{20,25}$ (Supplementary Video 1A, B). In this study, we performed a detailed quantitative analysis of the fluctuations in $\left[\mathrm{Ca}^{2+}\right]_{\mathrm{i}}$ using Fluo-4 $\mathrm{AM} \mathrm{Ca}^{2+}$ imaging and the FIBSI analysis program ${ }^{24}$ to further establish the nature of BDNF-induced changes in lamina II neurons of the SDH. Refer to Fig. $1 \mathrm{~A} 1-\mathrm{A} 3$ for an example of $\left[\mathrm{Ca}^{2+}\right]_{\mathrm{i}}$ fluctuations detected by the FIBSI program. Inspection of the FIBSI-processed recordings revealed a subset of naïve neurons, which were relatively 'silent' (Fig. 1B, left; raw recordings in Supplemental Fig. 1) displaying only small amplitude fluctuations for the duration of the recording ('silent' $=1.7 \pm 0.04 \mathrm{AU}$; naïve $=53.7 \pm 1.6 \mathrm{AU}$; $\mathrm{BDNF}=25.5 \pm 0.7 \mathrm{AU}$ ). No 'silent' neurons were found in the BDNF treatment group (Fig. 1B, right). Refer to Table 1 for detailed statistical information. This stark contrast suggested BDNF may be unmasking, or activating, these 'silent' neurons. We grouped the fluctuations in the 'silent' neurons together for further comparison to the fluctuations in the other active naïve neurons and BDNF-treated neurons. Log-transformed fluctuation amplitudes (Fig. 1C) and area under the curve (AUC; Fig. 1D) were larger in the active naïve population and in BDNF treated neurons compared to 'silent' neurons. Unexpectedly, both measures were significantly decreased in the BDNF condition compared to naive. A plausible explanation for this effect was that chronic BDNF induced a concomitant enhancement of $\left[\mathrm{Ca}^{2+}\right]_{\mathrm{i}}$ fluctuations in some neurons and depression in others. Indeed, the effects of BDNF on the relative frequencies (\%) for the fluctuation amplitudes (amplitudes were normalized to the largest fluctuation in each neuron) revealed a biphasic effect; frequencies of the smaller- and larger-amplitude fluctuations were greater in the BDNF-treated neurons compared to the naïve and 'silent' neurons (Fig. 1E). We next compared the mean parameters between neurons in order to control for differences in sampling periods. $\mathrm{A}<10 \%$ maximal fluctuation amplitude cut-off was applied to each neuron to reduce the effects of low-amplitude noise. As expected, nonparametric comparisons indicated that fluctuation amplitude (Fig. 1F) and AUC (Fig. 1G) were both greater in the naïve and BDNF-treated neurons compared to the 'silent' neurons, but were not significantly different between naïve and BDNF-treated neurons. Further analysis of the fluctuation kinetics indicated no effect on duration (Fig. $1 \mathrm{H}$ ), a modest, but significant decrease in rise time (i.e., peak time is more negative) compared to the 'silent' neurons (Fig. 1I), and no effect on frequency (Fig. 1J).

Octanol, a gap junction blocker, disinhibits a population of superficial dorsal horn neurons in BDNF-treated cultures. It has been shown that gap junctions have regulatory control over network activity in the substantia gelatinos $a^{26-29}$. Therefore, gap junctions may play a role in regulating the properties of $\left[\mathrm{Ca}^{2+}\right]_{\mathrm{i}}$ across the network, but the response of individual neurons to the chronic BDNF in the culture may be masked by the complex responses of the different types of neurons to $\mathrm{BDNF}^{20,30}$. To address this and determine the potential role of gap junctions in mediating the BDNF-induced fluctuations, we recorded $\left[\mathrm{Ca}^{2+}\right]_{\mathrm{i}}$ from SDH neurons exposed to chronic BDNF and then applied a non-specific gap junction blocker octanol at $1 \mathrm{mM}$ for $\geq 3 \mathrm{~min}$. We again performed a detailed analysis using the FIBSI program and $\mathrm{a}<10 \%$ maximal fluctuation amplitude cut-off was applied to each neuron. Inspection of the FIBSI-processed recordings revealed octanol disproportionately affected some neurons compared to others (i.e., large increases in amplitude and AUC in some neurons vs minor decreases in others). Neurons were grouped based on whether the octanol treatment caused a negative (decrease, $\mathrm{n}=39$ ) or positive (increase, $\mathrm{n}=40$ ) fold change in mean fluctuation amplitude compared to the BDNF (control) condition (Fig. 2A). Paired analyses indicated blocking gap junctions with octanol decreased the frequency of the BDNF-induced fluctuations (Fig. 2B) while increasing their duration (Fig. 2C) only in the neurons that had a positive fold change in mean fluctuation amplitude. Fluctuation rise time was also significantly decreased in the same group of neurons (Fig. 2D). These data suggest that blocking gap junctions with octanol selectively disinhibited the response to chronic BDNF in a subpopulation of SDH neurons.

Chronic BDNF induces synchrony of $\left[\mathrm{Ca}^{2+}\right]_{\mathrm{i}}$ fluctuations in organotypic cultures, and blocking gap junctions reduces BDNF-induced synchrony. Previous work has shown BDNF-treated SDH neurons generate synchronous $\left[\mathrm{Ca}^{2+}\right]_{\mathrm{i}}$ fluctuations ${ }^{20}$. We verified that finding by using the FIBSI-processed recordings and comparing the degree of synchrony between neurons imaged together in naive and BDNF-treated cultures. Adjacency matrixes were generated using Pearson $r$ coefficients; the recording of each neuron was correlated with every other neuron imaged within the same naïve or BDNF-treated culture using a Pearson cor- 

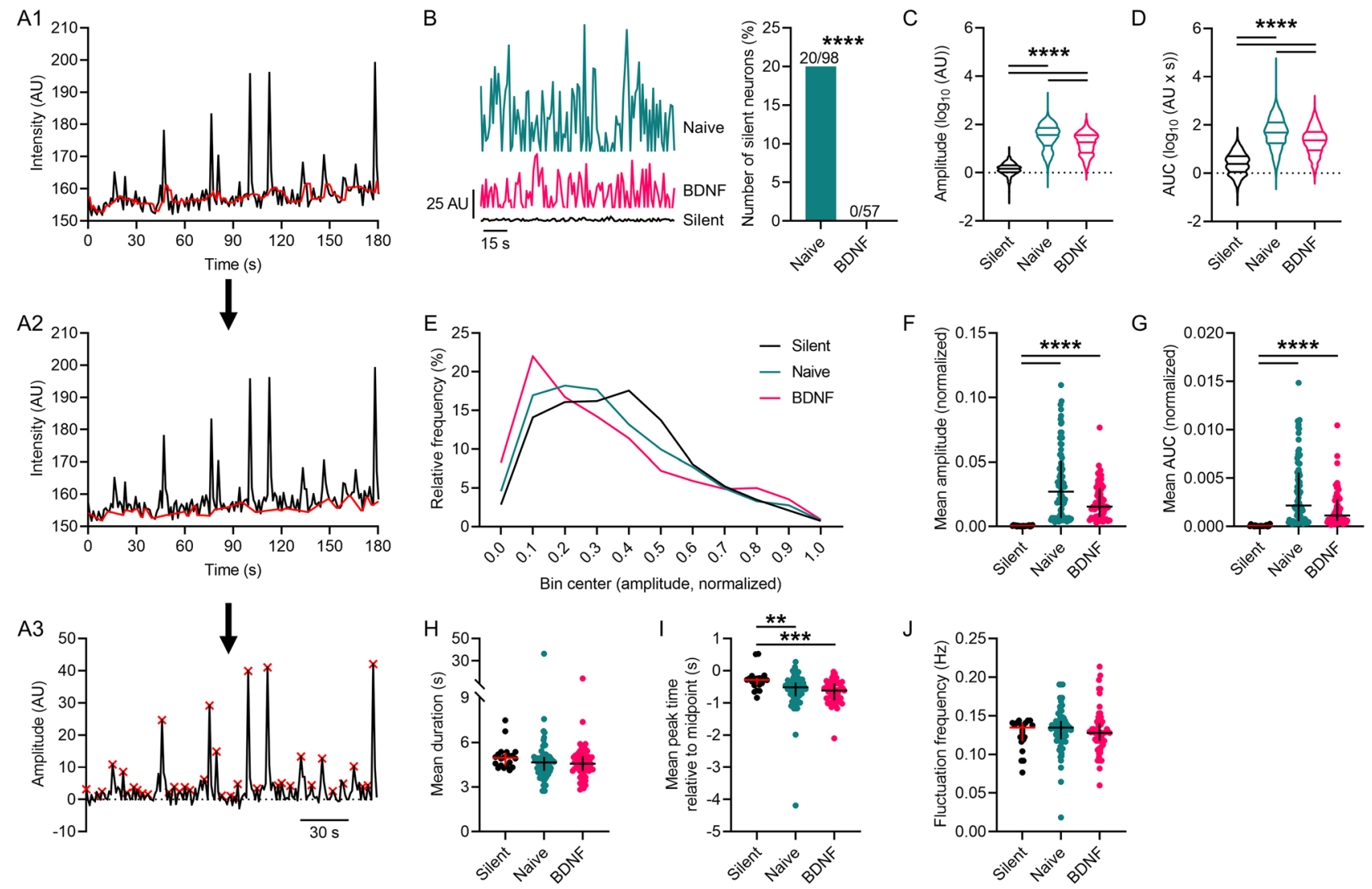

Figure 1. BDNF-induced fluctuations of $\left[\mathrm{Ca}^{2+}\right]_{\mathrm{I}}$ in superficial dorsal horn neurons. (A1-A3) Example recording $\left[\mathrm{Ca}^{2+}\right]_{\mathrm{i}}$ fluctuations sampled in a SDH neuron and the processing steps used by the FIBSI eventdetection program. The running median (red line in $\mathrm{A} 1$, window $=\sim 5 \mathrm{~s}$ ) was calculated based on the raw amplitude (AU) and time coordinate data, then the peaks below the running median were traced to form a reference line (red line in A2), and then the Ramer-Douglas-Peucker algorithm detected waveforms above the reference (red $\mathrm{x}$ at event peaks). (B) Left: Examples of $\left[\mathrm{Ca}^{2+}\right]_{\mathrm{i}}$ activity detected by FIBSI in 3 different neurons. Right: The proportion of 'silent' neurons in the BDNF-treatment condition was significantly reduced compared to the naïve condition. Proportions were compared using a Fisher exact test. (C, D) Violin plots of the logtransformed values for fluctuation amplitude and AUC. Fluctuation amplitudes and AUC were significantly increased in naïve and BDNF-treated neurons compared to the 'silent' neurons, while both measures in the BDNF-treated neurons were significantly reduced compared to naïve. Neurons sampled: 'silent' $\mathrm{n}=20$ (849 fluctuations), naïve $n=78$ (2651 fluctuations), and BDNF $n=57$ (1555 fluctuations). Median with quartiles shown. Comparisons made using Brown-Forsythe and Welch's ANOVA tests and Games-Howell post-hoc test. (E) Relative frequency (\%) of the fluctuations binned by amplitude. Amplitude values were normalized to the largest fluctuation in each neuron. (F-G) The mean fluctuation amplitude and AUC in the naïve and BDNFtreated neurons were significantly increased compared to the 'silent' neurons. (H-J) Further analysis revealed little to no effect of BDNF on fluctuation duration or frequency, but the fluctuations in 'silent' neurons exhibited longer rise times. For scatterplots F-J, the fluctuations that were $<10 \%$ the maximal fluctuation amplitude in each neuron were omitted to reduce the effects of low-amplitude noise. Scatterplots in F-J show the median with the interquartile range. All comparisons of medians in F-K were made using Kruskal-Wallis tests and Dunn's post-hoc test. ${ }^{* *} P<0.01,{ }^{* * *} P<0.001,{ }^{* * *} P<0.0001$.

relation matrix. The adjacency matrixes for the BDNF-treated cultures showed the neurons were more positively correlated with Pearson $r$ coefficients closer to 1 (Fig. 3A), suggesting they exhibited more synchronous $\left[\mathrm{Ca}^{2+}\right]_{i}$ fluctuations than the naïve cultures. Indeed, we observed synchronous spikes in $\left[\mathrm{Ca}^{2+}\right]_{\mathrm{i}}$ across multiple BDNFtreated neurons compared to the naïve neurons (Fig. 3B1-B2). Raster plots depicting the $\left[\mathrm{Ca}^{2+}\right]_{i}$ fluctuation peaks revealed the presence of multiple waves of synchronous activity in BDNF-treated neurons (Fig. 3C1-C2). To further confirm this prediction, the mean coefficient for each neuron was calculated (i.e., the mean correlation of each neuron with every other neuron in its respective culture; refer to Fig. 3A for Fisher Z transformation steps). Comparison of the mean Pearson $r$ coefficients revealed BDNF-treated neurons were more positively correlated with other neurons in the culture than naïve neurons (Fig. 3D). Considering the role gap junctions may play in regulating network activity and synchronous activity, this result led us to anticipate that blocking gap junctions may reduce BDNF-induced synchrony within the culture. We used the same approach to measure synchrony among neurons within the same cultures treated with BDNF prior to application of octanol, and then we asked whether blocking gap junctions caused a decrease in the mean Pearson $r$ coefficients. Indeed, octanol caused a net decrease in the mean Pearson $r$ coefficient (Fig. 3E1,E2). Closer inspection revealed octanol 


\begin{tabular}{|c|c|c|}
\hline Figure & Test & Post-hoc comparisons \\
\hline \multirow{2}{*}{$1 \mathrm{~B}$} & Fisher exact test & \multirow{2}{*}{$n / a$} \\
\hline & Naïve vs BDNF: $\mathrm{P}<0.0001$ & \\
\hline \multirow{4}{*}{$1 \mathrm{C}$} & Brown-Forsythe & Games-Howell's multiple comparison test \\
\hline & $\mathrm{F}_{(2,4598)}=3373, \mathrm{P}<0.0001$ & \multirow{3}{*}{$\begin{array}{l}\text { Silent vs naïve: } \mathrm{P}<0.0001 \text {; silent vs BDNF: } \mathrm{P}<0.0001 \text {; naïve vs } \\
\text { BDNF: } \mathrm{P}<0.0001\end{array}$} \\
\hline & \begin{tabular}{|l|} 
Welch's ANOVA \\
\end{tabular} & \\
\hline & $\mathrm{W}_{(2,2892)}=5458, \mathrm{P}<0.0001$ & \\
\hline \multirow{4}{*}{$1 \mathrm{D}$} & Brown-Forsythe & Games-Howell's multiple comparison test \\
\hline & $\mathrm{F}_{(2,4280)}=1737, \mathrm{P}<0.0001$ & \multirow{3}{*}{$\begin{array}{l}\text { Silent vs naïve: } \mathrm{P}<0.0001 \text {; silent vs BDNF: } \mathrm{P}<0.0001 \text {; naïve vs } \\
\text { BDNF: } \mathrm{P}<0.0001\end{array}$} \\
\hline & Welch's ANOVA & \\
\hline & $\mathrm{W}_{(2,2502)}=2032, \mathrm{P}<0.0001$ & \\
\hline \multirow[b]{2}{*}{$1 \mathrm{~F}$} & Kruskal-Wallis test & Dunn's multiple comparison test \\
\hline & $\mathrm{KW}=54.23, \mathrm{P}<0.0001$ & $\begin{array}{l}\text { Silent vs naïve: } \mathrm{P}<0.0001 \text {; silent vs } \mathrm{BDNF} \text { : } \mathrm{P}<0.0001 \text {; naïve vs } \\
\text { BDNF: } \mathrm{P}=0.0939\end{array}$ \\
\hline \multirow[b]{2}{*}{$1 \mathrm{G}$} & Kruskal-Wallis test & Dunn's multiple comparison test \\
\hline & $\mathrm{KW}=54.35, \mathrm{P}<0.0001$ & $\begin{array}{l}\text { Silent vs naïve: } \mathrm{P}<0.0001 \text {; silent vs } \mathrm{BDNF} \text { : } \mathrm{P}<0.0001 \text {; naïve vs } \\
\text { BDNF: } \mathrm{P}=0.0732\end{array}$ \\
\hline \multirow{2}{*}{$1 \mathrm{H}$} & Kruskal-Wallis test & \multirow{2}{*}{$n / a$} \\
\hline & $\mathrm{KW}=4.507, \mathrm{P}=0.1051$ & \\
\hline \multirow[b]{2}{*}{$1 \mathrm{I}$} & Kruskal-Wallis test & Dunn's multiple comparison test \\
\hline & $\mathrm{KW}=15.04, \mathrm{P}=0.0005$ & $\begin{array}{l}\text { Silent vs naïve: } \mathrm{P}=0.0087 \text {; silent vs } \mathrm{BDNF}: \mathrm{P}=0.0003 \text {; naïve vs } \\
\text { BDNF: } \mathrm{P}=0.4002\end{array}$ \\
\hline \multirow{2}{*}{$1 \mathrm{~J}$} & Kruskal-Wallis test & \multirow{2}{*}{$n / a$} \\
\hline & $\mathrm{KW}=2.810, \mathrm{P}=0.2454$ & \\
\hline \multirow{5}{*}{$2 \mathrm{~B}$} & Two-way RM ANOVA & Sidak's multiple comparison test \\
\hline & Interaction: $\mathrm{F}_{(1,77)}=29.27, \mathrm{P}<0.0001,10.65 \%$ total variation & \multirow{4}{*}{$\begin{array}{l}\text { Control vs octanol (Decrease): } \mathrm{P}=0.4469 \text {; Control vs octanol } \\
\text { (Increase): } \mathrm{P}<0.0001\end{array}$} \\
\hline & Group effect: $\mathrm{F}_{(1,77)}=0.0392, \mathrm{P}=0.8437,0.028 \%$ total variation & \\
\hline & $\begin{array}{l}\text { Treatment effect: } \mathrm{F}_{(1,77)}=14.31, \mathrm{P}=0.0003,5.208 \% \text { total vari- } \\
\text { ation }\end{array}$ & \\
\hline & $\begin{array}{l}\text { Subjects (matching) effect: } \mathrm{F}_{(77,77)}=1.994, \mathrm{P}=0.0014,55.89 \% \\
\text { total variation }\end{array}$ & \\
\hline \multirow{5}{*}{$2 \mathrm{C}$} & Two-way RM ANOVA & Sidak's multiple comparison test \\
\hline & Interaction: $\mathrm{F}_{(1,77)}=13.95, \mathrm{P}=0.0004,7.126 \%$ total variation & \multirow{4}{*}{$\begin{array}{l}\text { Control vs octanol (Decrease): } \mathrm{P}=0.9638 \text {; Control vs octanol } \\
\text { (Increase): } \mathrm{P}<0.0001\end{array}$} \\
\hline & Group effect: $\mathrm{F}_{(1,77)}=7.287, \mathrm{P}=0.0085,4.105 \%$ total variation & \\
\hline & $\begin{array}{l}\text { Treatment effect: } \mathrm{F}_{(1,77)}=11.50, \mathrm{P}=0.0011,5.875 \% \text { total vari- } \\
\text { ation }\end{array}$ & \\
\hline & $\begin{array}{l}\text { Subjects (matching) effect: } \mathrm{F}_{(77,77)}=1.102, \mathrm{P}=0.3349,43.38 \% \\
\text { total variation }\end{array}$ & \\
\hline \multirow{5}{*}{$2 \mathrm{D}$} & Two-way RM ANOVA & Sidak's multiple comparison test \\
\hline & Interaction: $\mathrm{F}_{(1,77)}=13.63, \mathrm{P}=0.0004,7.764 \%$ total variation & \multirow{4}{*}{$\begin{array}{l}\text { Control vs octanol (Decrease): } \mathrm{P}=0.1876 \text {; Control vs octanol } \\
\text { (Increase): } \mathrm{P}=0.0013\end{array}$} \\
\hline & Group effect: $\mathrm{F}_{(1,77)}=0.080, \mathrm{P}=0.7780,0.049 \%$ total variation & \\
\hline & $\begin{array}{l}\text { Treatment effect: } \mathrm{F}_{(1,77)}=1.725, \mathrm{P}=0.1929,0.983 \% \text { total vari- } \\
\text { ation }\end{array}$ & \\
\hline & $\begin{array}{l}\text { Subjects (matching) effect: } \mathrm{F}_{(77,77)}=1.078, \mathrm{P}=0.3709,47.28 \% \\
\text { total variation }\end{array}$ & \\
\hline \multirow{2}{*}{$3 \mathrm{D}$} & Mann-Whitney test & \multirow{2}{*}{$n / a$} \\
\hline & $\mathrm{U}=606.5, \mathrm{P}=0.0010$ & \\
\hline \multirow{2}{*}{$3 \mathrm{E} 1$} & Wilcoxon matched-pairs signed rank test & \multirow{2}{*}{$n / a$} \\
\hline & $\mathrm{W}=-1373, \mathrm{P}<0.0001$ & \\
\hline \multirow{5}{*}{$3 \mathrm{E} 2$} & Two-way RM ANOVA & Sidak's multiple comparison test \\
\hline & Interaction: $\mathrm{F}_{(1,58)}=37.70, \mathrm{P}<0.0001,6.086 \%$ total variation & \multirow{4}{*}{$\begin{array}{l}\text { Control vs octanol (Decrease): } \mathrm{P}<0.0001 \text {; Control vs octanol } \\
\text { (Increase): } \mathrm{P}=0.0450\end{array}$} \\
\hline & Group effect: $\mathrm{F}_{(1,58)}=38.23, \mathrm{P}<0.0001,29.22 \%$ total variation & \\
\hline & $\begin{array}{l}\text { Treatment effect: } \mathrm{F}_{(1,58)}=83.93, \mathrm{P}<0.0001,13.55 \% \text { total vari- } \\
\text { ation }\end{array}$ & \\
\hline & $\begin{array}{l}\text { Subjects (matching) effect: } \mathrm{F}_{(58,58)}=4.736, \mathrm{P}<0.0001,44.34 \% \\
\text { total variation }\end{array}$ & \\
\hline
\end{tabular}




\begin{tabular}{|c|c|c|}
\hline Figure & Test & Post-hoc comparisons \\
\hline \multirow{12}{*}{$4 \mathrm{~A}-\mathrm{B}$} & Paired $\mathrm{t}$ tests & \multirow{12}{*}{$n / a$} \\
\hline & Control vs $0 \mathrm{Ca}^{2+}$ : Amplitude $\mathrm{P}>0.05$; Frequency $\mathrm{P}>0.05$ & \\
\hline & Control vs $\mathrm{Cd}^{2+}$ : Amplitude $\mathrm{P}>0.05$; Frequency $\mathrm{P}>0.05$ & \\
\hline & Control vs $\mathrm{Ni}^{2+}$ : Amplitude $\mathrm{P}>0.05$; Frequency $\mathrm{P}<0.001$ & \\
\hline & $\begin{array}{l}\text { Control vs nitrendipine: Amplitude } \mathrm{P}>0.05 \text {; Frequency } \\
\mathrm{P}<0.001\end{array}$ & \\
\hline & $\begin{array}{l}\text { Control vs } \omega \text {-conotoxin: Amplitude } \mathrm{P}>0.05 \text {; Frequency } \\
\mathrm{P}<0.001\end{array}$ & \\
\hline & Control vs TTX: Amplitude P >0.05; Frequency $\mathrm{P}>0.05$ & \\
\hline & Control vs riluzole: Amplitude $\mathrm{P}>0.05$; Frequency $\mathrm{P}<0.001$ & \\
\hline & Control vs NBQX: Amplitude P >0.05; Frequency P $>0.05$ & \\
\hline & Control vs D-AP5: Amplitude P $<0.001$; Frequency $\mathrm{P}>0.05$ & \\
\hline & $\begin{array}{l}\text { Control vs kynurenic acid: Amplitude } \mathrm{P}>0.05 \text {; Frequency } \\
\mathrm{P}>0.05\end{array}$ & \\
\hline & Control vs GABA: Amplitude $\mathrm{P}>0.05$; Frequency $\mathrm{P}>0.05$ & \\
\hline \multirow{4}{*}{$5 \mathrm{C} 2$} & Brown-Forsythe & Games-Howell's multiple comparison test \\
\hline & $\mathrm{F}_{(3,2916)}=311.1, \mathrm{P}<0.0001$ & \multirow{3}{*}{$\begin{array}{l}\text { Control vs BDNF (Delay): } \mathrm{P}=0.4264 \text {; Control vs BDNF (Tonic) } \\
\mathrm{P}<0.0001\end{array}$} \\
\hline & Welch's ANOVA & \\
\hline & $\mathrm{W}_{(3,2862)}=504.2, \mathrm{P}<0.0001$ & \\
\hline \multirow{4}{*}{$5 \mathrm{D} 2$} & Brown-Forsythe & Games-Howell's multiple comparison test \\
\hline & $\mathrm{F}_{(3,1761)}=419.3, \mathrm{P}<0.0001$ & \multirow{3}{*}{$\begin{array}{l}\text { Control vs BDNF (Delay): } \mathrm{P}<0.0001 \text {; Control vs BDNF (Tonic) } \\
\mathrm{P}<0.0001\end{array}$} \\
\hline & Welch's ANOVA & \\
\hline & $\mathrm{W}_{(3,2791)}=541.8, \mathrm{P}<0.0001$ & \\
\hline \multirow{4}{*}{500} & Brown-Forsythe & Games-Howell's multiple comparison test \\
\hline & $\mathrm{F}_{(3,2550)}=159.1, \mathrm{P}<0.0001$ & \multirow{3}{*}{$\begin{array}{l}\text { Control vs BDNF (Delay): } \mathrm{P}<0.0001 \text {; Control vs BDNF (Tonic) } \\
\mathrm{P}=0.0012\end{array}$} \\
\hline & Welch's ANOVA & \\
\hline & $\mathrm{W}_{(3,2759)}=162.3, \mathrm{P}<0.0001$ & \\
\hline \multirow{4}{*}{$5 \mathrm{~F} 2$} & Brown-Forsythe & Games-Howell's multiple comparison test \\
\hline & $\mathrm{F}_{(3,2027)}=14.5, \mathrm{P}<0.0001$ & \multirow{3}{*}{$\begin{array}{l}\text { Control vs BDNF (Delay): } \mathrm{P}=0.1284 \text {; Control vs BDNF (Tonic) } \\
\mathrm{P}<0.0001\end{array}$} \\
\hline & Welch's ANOVA & \\
\hline & $\mathrm{W}_{(3,2860)}=27.93, \mathrm{P}<0.0001$ & \\
\hline \multirow{6}{*}{ S4A2 } & Kruskal-Wallis test & Dunn's multiple comparison test \\
\hline & $\mathrm{KW}=44.87, \mathrm{P}<0.0001$ & $\begin{array}{l}\text { Silent vs naïve: } \mathrm{P}<0.0001 \text {; silent vs } \mathrm{BDNF}: \mathrm{P}=0.0002 \text {; naïve vs } \\
\text { BDNF: } \mathrm{P}=0.0020\end{array}$ \\
\hline & Brown-Forsythe & Games-Howell's multiple comparison test \\
\hline & $\mathrm{F}_{(3,71793)}=1122, \mathrm{P}<0.0001$ & \multirow{3}{*}{$\begin{array}{l}\text { Control vs BDNF (Delay): } \mathrm{P}<0.0001 \text {; Control vs BDNF (Tonic) } \\
\mathrm{P}<0.0001\end{array}$} \\
\hline & Welch's ANOVA & \\
\hline & $\mathrm{W}_{(3,54420)}=2011, \mathrm{P}<0.0001$ & \\
\hline \multirow{4}{*}{ S4A4 } & Brown-Forsythe & Games-Howell's multiple comparison test \\
\hline & $\mathrm{F}_{(3,29988)}=851.4, \mathrm{P}<0.0001$ & \multirow{3}{*}{$\begin{array}{l}\text { Control vs BDNF (Delay): } \mathrm{P}<0.0001 \text {; Control vs BDNF (Tonic) } \\
\mathrm{P}<0.0001\end{array}$} \\
\hline & Welch's ANOVA & \\
\hline & $\mathrm{W}_{(3,18137)}=2294, \mathrm{P}<0.0001$ & \\
\hline \multirow{4}{*}{ S4B2 } & Brown-Forsythe & Games-Howell's multiple comparison test \\
\hline & $\mathrm{F}_{(3,55836)}=870.5, \mathrm{P}<0.0001$ & \multirow{3}{*}{$\begin{array}{l}\text { Control vs BDNF (Delay): } \mathrm{P}<0.0001 \text {; Control vs BDNF (Tonic) } \\
\mathrm{P}<0.0001\end{array}$} \\
\hline & Welch's ANOVA & \\
\hline & $\mathrm{W}_{(3,54028)}=850.3, \mathrm{P}<0.0001$ & \\
\hline \multirow{4}{*}{ S4B4 } & Brown-Forsythe & Games-Howell's multiple comparison test \\
\hline & $\mathrm{F}_{(3,22611)}=438.9, \mathrm{P}<0.0001$ & \multirow{3}{*}{$\begin{array}{l}\text { Control vs BDNF (Delay): } \mathrm{P}<0.0001 \text {; Control vs BDNF (Tonic) } \\
\mathrm{P}<0.0001\end{array}$} \\
\hline & Welch's ANOVA & \\
\hline & $\mathrm{W}_{(3,17636)}=1298, \mathrm{P}<0.0001$ & \\
\hline \multirow{4}{*}{ S4C2 } & Brown-Forsythe & Games-Howell's multiple comparison test \\
\hline & $\mathrm{F}_{(3,72912)}=203.0, \mathrm{P}<0.0001$ & \multirow{3}{*}{$\begin{array}{l}\text { Control vs BDNF (Delay): } \mathrm{P}<0.0001 \text {; Control vs BDNF (Tonic): } \\
\mathrm{P}<0.0001\end{array}$} \\
\hline & Welch's ANOVA & \\
\hline & $\mathrm{W}_{(3,51340)}=169.4, \mathrm{P}<0.0001$ & \\
\hline & Brown-Forsythe & Games-Howell's multiple comparison test \\
\hline & $\mathrm{F}_{(3,26038)}=26.33, \mathrm{P}<0.0001$ & \\
\hline S4C4 & Welch's ANOVA & $\begin{array}{l}\text { Control vs BDNF (Delay): } \mathrm{P}=0.0062 \text {; Control vs } \mathrm{BDNF} \text { (Tonic): } \\
\mathrm{P}=0.6416\end{array}$ \\
\hline & $\mathrm{W}_{(3,14707)}=27.89, \mathrm{P}<0.0001$ & \\
\hline
\end{tabular}




\begin{tabular}{|c|c|c|}
\hline Figure & Test & Post-hoc comparisons \\
\hline \multirow{4}{*}{ S4D2 } & Brown-Forsythe & Games-Howell's multiple comparison test \\
\hline & $\mathrm{F}_{(3,76383)}=171.1, \mathrm{P}<0.0001$ & \multirow{3}{*}{$\begin{array}{l}\text { Control vs BDNF (Delay): } \mathrm{P}<0.0001 \text {; Control vs BDNF (Tonic): } \\
\mathrm{P}<0.0001\end{array}$} \\
\hline & Welch's ANOVA & \\
\hline & $\mathrm{W}_{(3,51987)}=167.3, \mathrm{P}<0.0001$ & \\
\hline \multirow{4}{*}{ S4D4 } & Brown-Forsythe & Games-Howell's multiple comparison test \\
\hline & $\mathrm{F}_{(3,26111)}=62.09, \mathrm{P}<0.0001$ & \multirow{3}{*}{$\begin{array}{l}\text { Control vs BDNF (Delay): } \mathrm{P}<0.0001 \text {; Control vs BDNF (Tonic): } \\
\mathrm{P}<0.0001\end{array}$} \\
\hline & Welch's ANOVA & \\
\hline & $\mathrm{W}_{(3,16008)}=62.78, \mathrm{P}<0.0001$ & \\
\hline
\end{tabular}

Table 1. Detailed results of statistical analysis per figure.

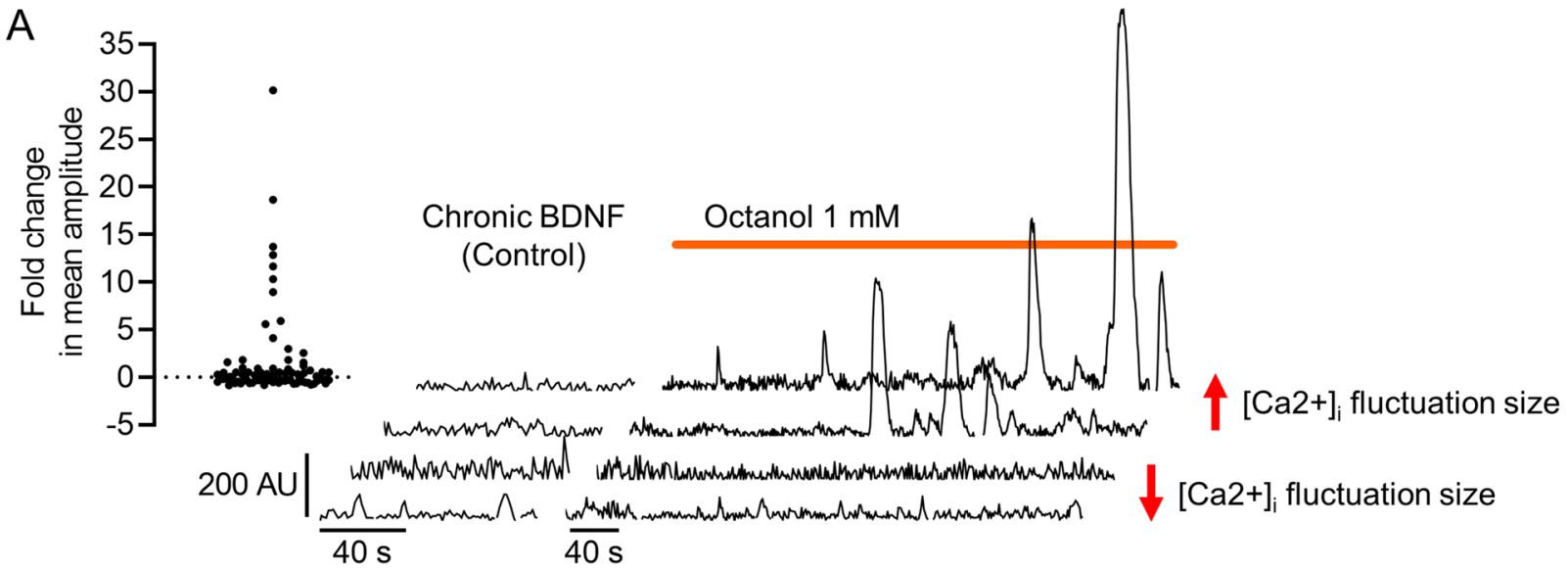

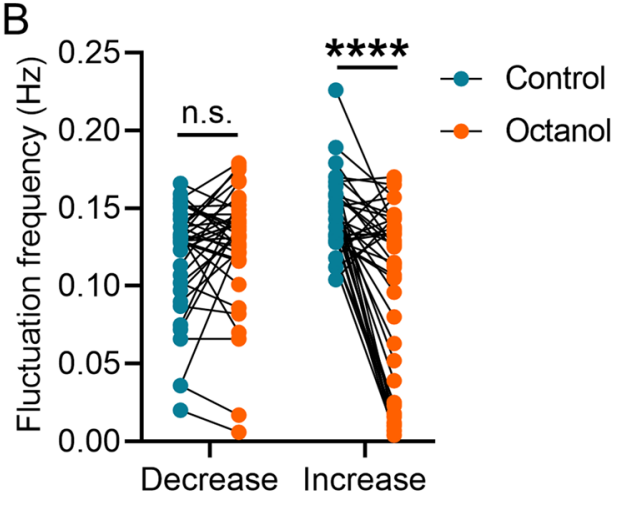

Fold change in mean amplitude

C

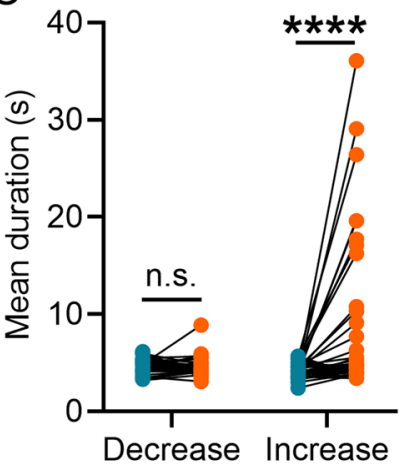

Fold change in mean amplitude

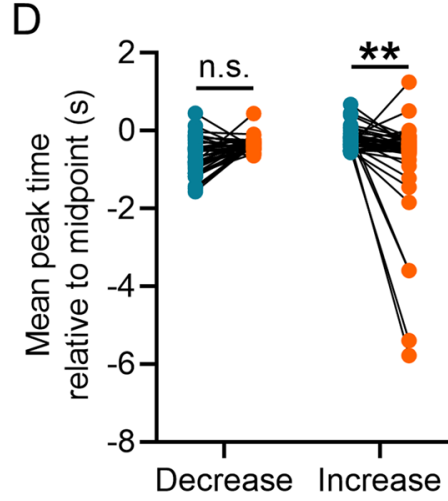

Fold change in mean amplitude

Figure 2. Effect of gap junction blocker octanol on BDNF-induced $\left[\mathrm{Ca}^{2+}\right]_{\mathrm{I}}$ fluctuations. (A) Scatterplot summarizing the effects of octanol on mean fluctuation amplitude and examples of FIBSI-processed recordings. The two bottom recordings show a decrease in fluctuation amplitude and the two top show an increase. (B-D) Neurons were sorted based on whether they exhibited a positive or negative fold change in mean fluctuation amplitude in response to octanol. Paired analyses showed octanol selectively and significantly decreased the mean fluctuation frequency, increased the mean fluctuation duration, and decreased the mean fluctuation rise time (i.e., more negative peak time) in the neurons with positive fold changes in mean fluctuation amplitude. Control vs octanol paired comparisons for the groups in (B-D) were made using two-way repeated measures ANOVAs and Sidak's post-hoc test. ${ }^{* *} P<0.01,{ }^{* * *} P<0.0001$.

decreased the mean Pearson $r$ coefficient in $100 \%$ (25/25) of the neurons in the group with decreased fluctuation amplitudes, while only $63 \%$ (22/35) of the neurons in the group with increased fluctuation amplitudes exhibited a decreased mean Pearson $r$ coefficient. This suggests that blocking gap junctions may actually increase $\left[\mathrm{Ca}^{2+}\right]_{\mathrm{i}}$ fluctuation synchrony in some neurons. 
A

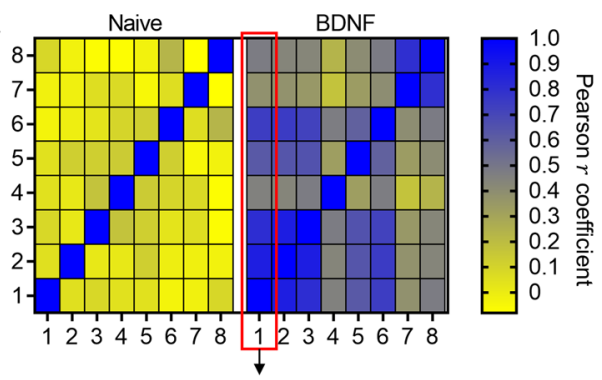

Pearson $r$ coefficient for neuron $i_{1,2}, i_{1,3}, \ldots, i_{1, n}$ $\downarrow$

Fisher Z: $z^{\prime}=0.5[\ln (1+r)-\ln (1-r)]$

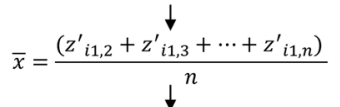

Back transform using Fisher Z

$\downarrow$

Mean Pearson $r$ for $i_{1}$, repeat for $i_{2}, i_{3}, \ldots, i_{n}$
B1 Naïve neurons

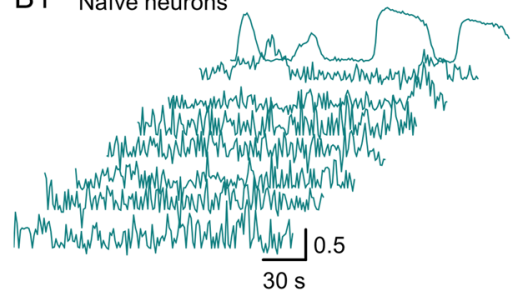

C1 Naïve neurons - fluctuation peaks
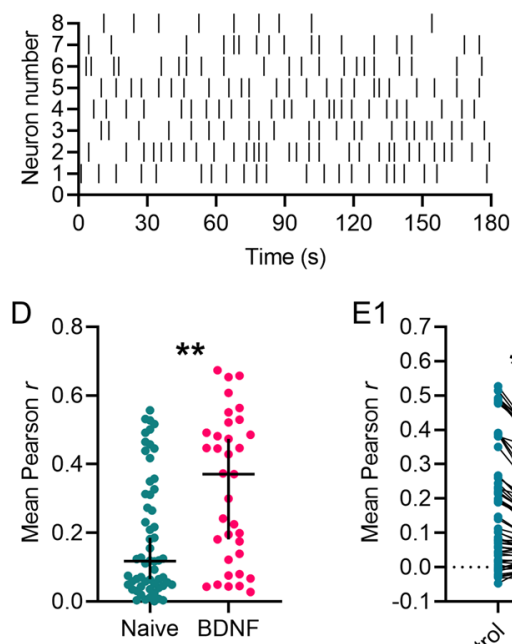

B2

BDNF-treated neurons

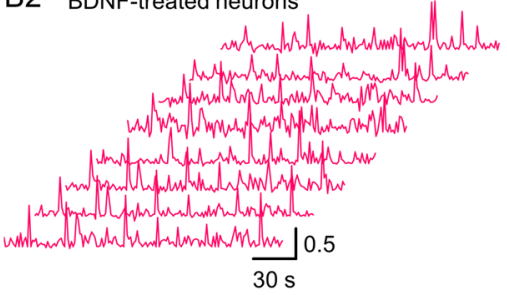

C2 BDNF-treated neurons - fluctuation peaks
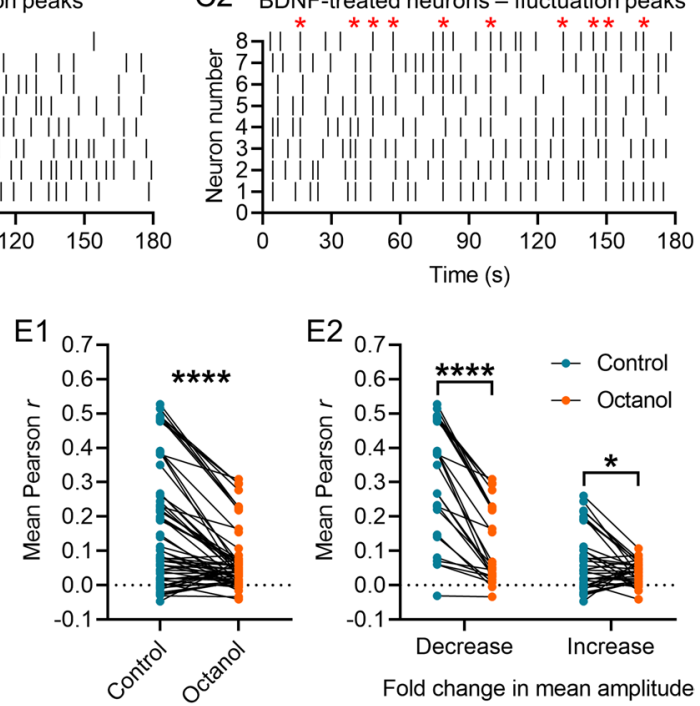

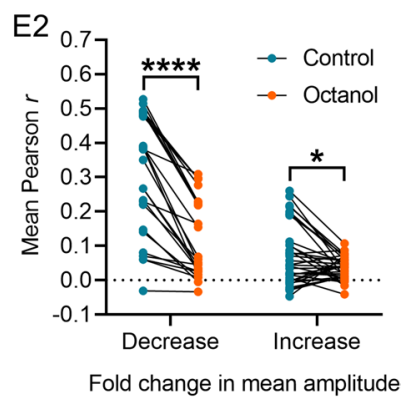

Figure 3. Synchrony of $[\mathrm{Ca} 2+]_{\mathrm{i}}$ fluctuations in BDNF-treated cultures and the effect of octanol. (A) Example adjacency matrix constructed from Pearson $r$ coefficients corresponding to the correlation in activity between neurons in a naïve or BDNF-treated culture, each with 8 sampled neurons. Steps to calculate the mean Pearson $r$ for each neuron within a culture using the Fisher $\mathrm{Z}$ transformation are also depicted. (B1,B2) Example recordings of asynchronous $\left[\mathrm{Ca}^{2+}\right]_{\mathrm{i}}$ fluctuations in naïve neurons and synchronous $\left[\mathrm{Ca}^{2+}\right]_{\mathrm{i}}$ fluctuations in BDNFtreated neurons. Recordings shown were processed by FIBSI for event detection and each one was normalized ( 0 to 1 scale) before calculation of the adjacency matrix in A. $(\mathbf{C 1}, \mathbf{C 2})$ Raster plots depicting the fluctuation peak times detected by FIBSI for the recordings in B1-B2. Red stars in C2 denote the presence of synchronous waves of $\left[\mathrm{Ca}^{2+}\right]_{\mathrm{i}}$ activity. (D) Neurons sampled from BDNF-treated cultures exhibited significantly greater mean Pearson $r$ coefficients compared to neurons sampled from naïve cultures. Neurons were imaged from 4 naïve cultures and 3 BDNF-treated cultures, each with $\geq 8$ neurons imaged. Medians shown with the $95 \%$ confidence interval. Medians were compared using a Mann-Whitney test. (E1,E2) Treatment with octanol significantly decreased the mean Pearson $r$ in neurons sampled from cultures exposed to chronic BDNF, and this treatment effect impacted both subsets of neurons binned based on their initial response to octanol. Neurons were imaged from 4 cultures. The control vs octanol paired comparison in $\mathrm{C} 1$ was made using a Wilcoxon rank sum test. Control vs octanol paired comparisons for the groups in C2 were made using a measures two-way repeated measures ANOVA and Sidak's post-hoc test. ${ }^{\star} P<0.05,{ }^{\star *} P<0.01,{ }^{\star * \star \star} P<0.0001$.

Pharmacology of $\left[\mathrm{Ca}^{2+}\right]_{i}$ fluctuations in BDNF-treated slices. In order to further characterize the properties of the $\left[\mathrm{Ca}^{2+}\right]_{\mathrm{i}}$ fluctuations, we used pharmacological treatments to block a variety of voltage-gated ion channels, glutamate receptors, and GABA receptors. Comparisons between the pharmacologically-treated neurons and the BDNF-treated (control) neurons revealed the $\left[\mathrm{Ca}^{2+}\right]_{\mathrm{i}}$ fluctuation amplitudes (Fig. $4 \mathrm{~A}$ ) and frequency (Fig. 4B) are controlled by diverse mechanisms (example recordings and data available in Supplemental Fig. 2). The fluctuations were ablated when extracellular $\mathrm{Ca}^{2+}$ was removed, when all voltage-gated calcium channels (VGCCs) were blocked with $\mathrm{Cd}^{2+}$, when all TTX-sensitive voltage-gated sodium channels (VGSCs) were blocked with tetrodotoxin (TTX), when AMPA or kainite glutamate receptors were blocked with CNQX/NBQX, and when GABA was applied to the cultures. Interestingly, the fluctuations remained when we blocked T-type (with $\mathrm{Ni}^{2+}$ ), L-type (with nitrendipine), or $\mathrm{N}$-type (with $\omega$-conotoxin) $\mathrm{Ca}^{2+}$ channels, although the frequency was significantly reduced with no effect on amplitude. Riluzole, which blocks glutamate release and VGSCs, only affected the fluctuation frequency but not amplitude. The NMDA blocker D-AP5 was the only blocker to significantly reduce fluctuation amplitudes, but it had no effect on frequency. We also noted that applying GABA-A blockers bicuculline $(10 \mu \mathrm{M})$ and strychnine $(1 \mu \mathrm{M})$ to BDNF-treated slices (Supplementary Fig. 2P) abolishes higher frequency smaller amplitude fluctuations, leaving inducing the larger amplitude low frequency fluctuations that occur in naive cultures (Supplementary Fig. 2P). These data suggest the BDNF-induced $\left[\mathrm{Ca}^{2+}\right]_{\mathrm{i}}$ fluctuations are controlled by a combination of VGCCs, TTX-sensitive VGSCs, GABA and NMDA receptors. 

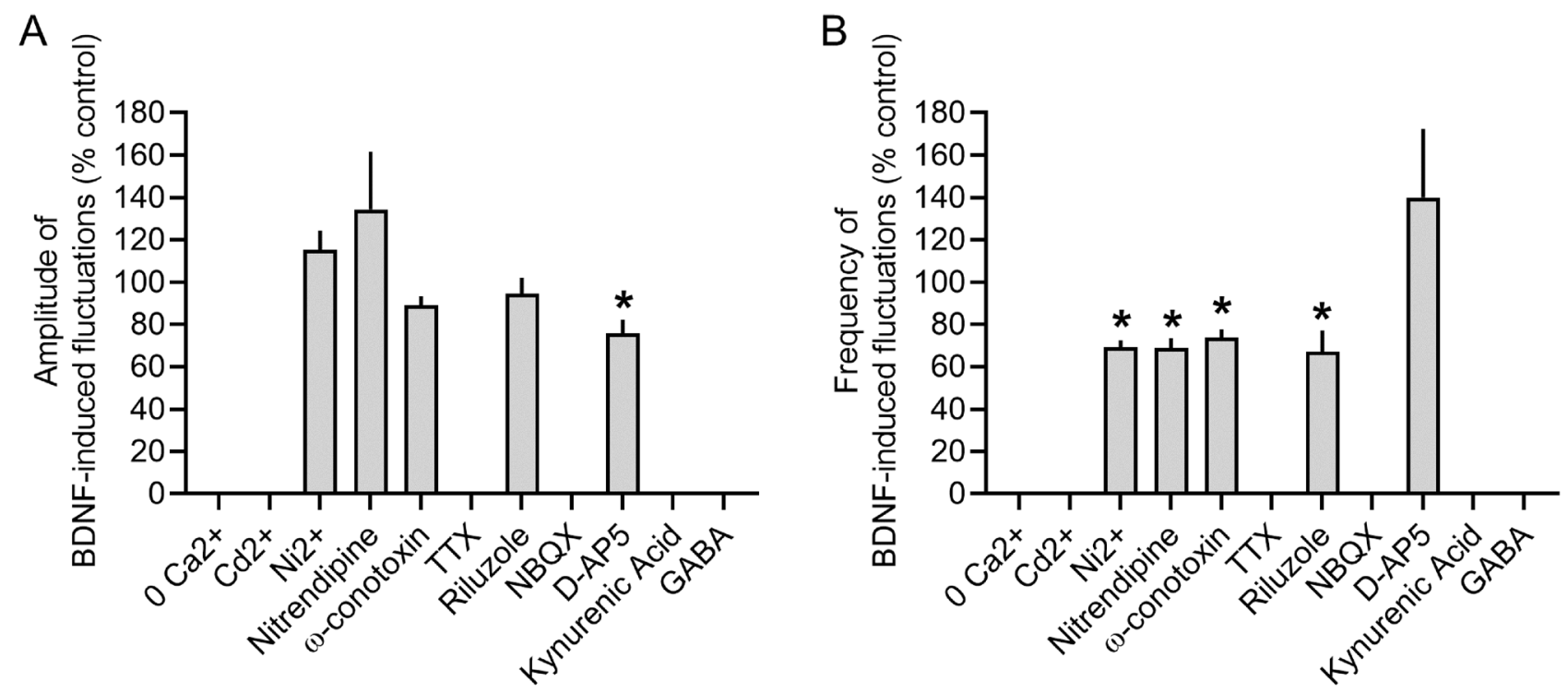

Figure 4. Pharmacology of $\left[\mathrm{Ca}^{2+}\right]_{\mathrm{i}}$ fluctuations from BDNF-treated cultures. Summary of the effects of various pharmacological treatments on the (A) amplitude and (B) frequency of the $\left[\mathrm{Ca}^{2+}\right]_{\mathrm{i}}$ fluctuations in BDNF-treated cultures compared to before drug treatment. Bars show the mean + standard error of the mean. ${ }^{\star} P<0.001$, paired t-test, $n=5-20$ cells per condition.

Clustering spontaneous excitatory postsynaptic currents (sEPSCs) reveals chronic BDNF treatment depresses excitability of delay and tonic-firing superficial dorsal horn neurons, mirroring the effect of BDNF on $\left[\mathrm{Ca}^{2+}\right]_{i}$ fluctuations. It is well established that BDNF release in the SDH is involved in neuropathic pain ${ }^{10,16,17}$. However, to our knowledge our findings are the first to show chronic BDNF treatment unmasks $\left[\mathrm{Ca}^{2+}\right]_{\mathrm{i}}$ fluctuations in a subpopulation of SDH neurons while depressing activity in others. We had previously shown that sEPSCs recorded from SDH neurons are perturbed in BDNF-treated spinal cord organotypic cultures ${ }^{20}$. It is plausible that the newly discovered unmasked BDNF-induced $\left[\mathrm{Ca}^{2+}\right]_{\mathrm{i}}$ fluctuations underlie changes in network excitability. We decided to readdress the effects of BDNF on network excitability by using the FIBSI program to quantitatively analyze a previous subset of sEPSC recordings in delay and tonic-firing SDH neurons (example recordings and low-pass filtering/matching methods are available in Supplemental Fig. 3). Inspection of the sEPSC interevent interval data indicated there might be natural clustering between single "small" sEPSCs and larger summated sEPSCs. We used partitioning around medoids (a medoid is like a centroid, but is restricted to an actual observation in the dataset) to cluster the sEPSCs in each neuron based on 3 sEPSC parameters: interevent interval (ms), amplitude (pA), and charge transfer ( $Q$; essentially area under the curve). Optimal clustering ( $\mathrm{k}$ medoids $=3-5$ per neuron) identified 3 main clusters of sEPSCs that were present in all delay (Fig. 5A1,A2) and tonic (Fig. 5B1,B2) neurons in both control and BDNF treatment conditions (clusters named based on amplitude; interevent interval): (1) small; short, (2) small; long, and (3) large. Two other clusters were identified (small; mid and medium), but they were not present in all neurons or treatment conditions and were not analyzed for this study. We were mainly interested in the effects of BDNF on the cluster of large-amplitude sEPSCs in both neuron types (refer to Supplemental Fig. 4 for the "small" sEPSC analysis). Chronic BDNF treatment significantly decreased sEPSC amplitudes (Fig. 5C1,C2) and charge transfer (Fig. 5D1,D2) in the tonic-firing neurons. We did not observe a significant effect on sEPSC amplitudes in the delay neurons, but charge transfer was significantly decreased. The sEPSCs in the BDNF-treated delay neurons were narrower (control duration $=83.6 \pm 1.2 \mathrm{~ms}$; BDNF duration $=49.9 \pm 0.4 \mathrm{~ms}$ ). The sEPSC interevent intervals were significantly shorter in both neuron types (Fig. 5E1,E2), and peak time measurements indicated BDNF significantly increased activation of the sEPSCs in the tonic neuron (Fig. 5F1,F2).

\section{Discussion}

The present study was undertaken to better understand mechanisms underlying synchronous activity among SDH neurons and the influence BDNF may have on network excitability in naïve, uninjured organotypic cultures. Our experiments indicate long-term exposure to BDNF produces a complex set of neuron-dependent changes in network excitability in the SDH. First, BDNF causes a concomitant activation of $\mathrm{Ca}^{2+}$ signaling in a subpopulation of 'silent' SDH neurons ( $20 \%$ sampled) and depression of $\mathrm{Ca}^{2+}$ signaling in many others. This finding was largely unexpected as we have previously shown chronic BDNF increases the size and frequency of $\left[\mathrm{Ca}^{2+}\right]_{\mathrm{i}}$ fluctuations ${ }^{20}$. It is plausible that the presence of the 'silent' neurons can greatly influence data analysis and interpretation depending on how they are grouped together, or discarded. Another possibility is that resolution differences between the two event-detection programs used (FIBSI in the current study, Mini Analysis Software (Synaptosoft, NJ)) in the previous study) could influence analysis of the $\left[\mathrm{Ca}^{2+}\right]_{\mathrm{i}}$ fluctuations. However, the goal of the present study was not to compare/contrast the two programs. The second significant finding was 

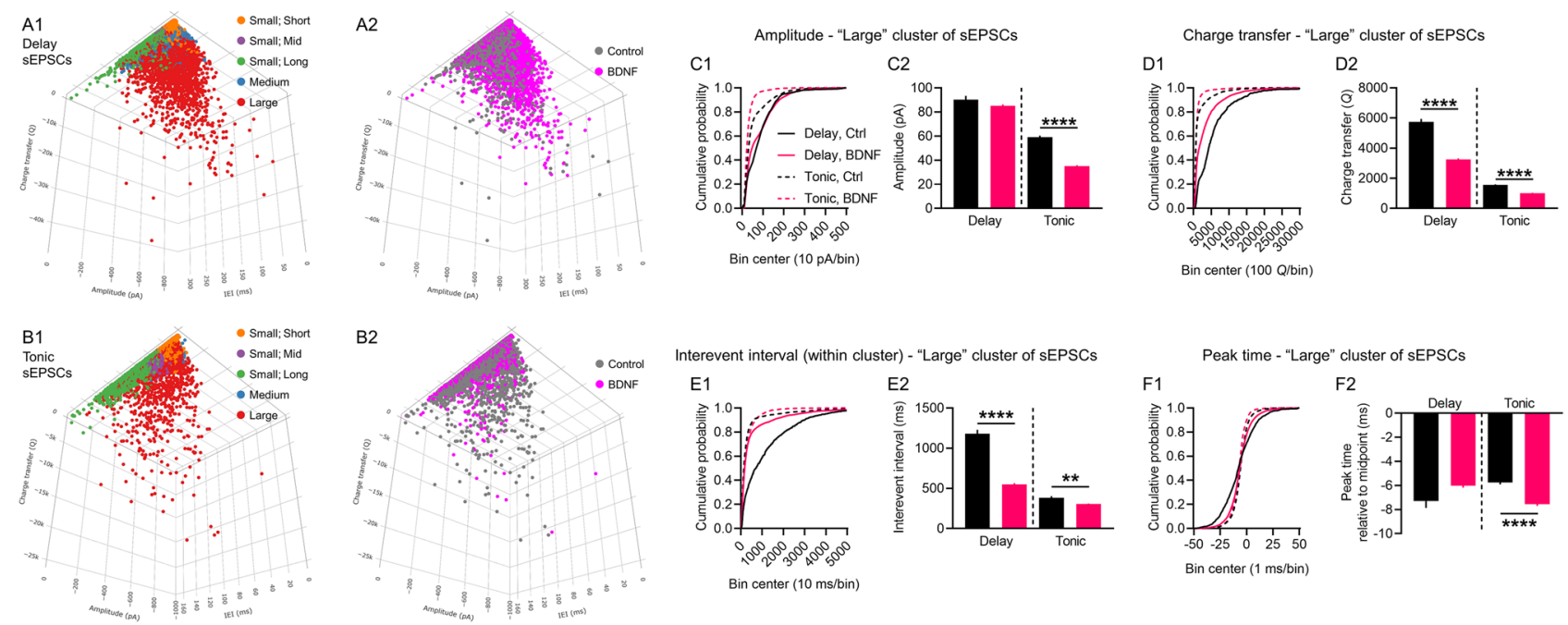

Figure 5. Cluster analysis of spontaneous EPSCs in delay and tonic-firing superficial dorsal horn neurons. Three dimensional representations of the sEPSCs in $(\mathbf{A 1}, \mathbf{A} 2)$ delay neurons and $(\mathbf{B 1}, \mathbf{B} 2)$ tonic neurons plotted based on interevent interval ( $\mathrm{x}$-axis), amplitude (y-axis), and charge transfer ( $\mathrm{z}$-axis). Mapping of the clustering results and treatment shown for the total sample of sEPSCs. The sEPSCs in each neuron were clustered independently from the other neurons. Optimal partitioning around medoids identified 3 primary clusters present in all neurons in both treatment conditions (naming based on amplitude; interevent interval): small; short, small; long, and large. Two additional clusters were identified in some neurons, but were not present in all neurons in both conditions: small; mid and medium. Neurons sampled: 19 delay ( 5 control, 14 BDNF); 9 tonic ( 5 control, 4 BDNF). Total number of sEPSCs: 33,206 in delay, control; 108,261 in delay, BDNF; 40,658 in tonic, control; 36,521 in tonic, BDNF. The effects of BDNF on sEPSC amplitude, charge transfer (i.e., area under the curve), and peak time were assessed for the 3 primary clusters (large cluster shown, refer to Supplemental Fig. 3 for the two small-amplitude clusters). (C1,C2) Treatment with BDNF significantly decreased sEPSC amplitudes in tonic neurons, but a significant effect was not observed in the delay neurons. Cumulative distributions of the measured amplitudes shown (left) alongside the means \pm standard error of the means (right). (D1,D2) The BDNF treatment significantly reduced sEPSC charge transfer measures in both delay and tonic neurons. (E1,E2) Interevent intervals for the sEPSCs within the large cluster were significantly decreased in the BDNF-treated neurons. (F1, F2) The sEPSC activation kinetics were significantly faster in the BDNF-treated neurons, but no significant effect was observed for the sEPSCs in the delay neurons. Large sEPSC cluster sample sizes: delay neurons control $n=753$, BDNF $n=4539$; tonic neurons control $n=2348$, BDNF $n=2367$. Comparisons between means were made using Brown-Forsythe and Welch's ANOVA tests and Games-Howell post-hoc test. ${ }^{* *} P<0.01$, ${ }^{* * * *} P<0.0001$.

that gap junction signaling may play a crucial role in regulating BDNF-induced changes in $\mathrm{Ca}^{2+}$ signaling in the SDH. Blockade of gap junctions with octanol unveiled a subpopulation of neurons that respond to BDNF with large-amplitude, low-frequency $\left[\mathrm{Ca}^{2+}\right]_{\mathrm{i}}$ fluctuations. This finding suggests that gap junctions modulate global network activity, either by directly or indirectly inhibiting those particular neurons with increased $\mathrm{Ca}^{2+}$ signaling. Indeed, this is supported by the third major finding that BDNF profoundly increases synchrony of the $[\mathrm{Ca} 2+]_{\mathrm{i}}$ fluctuations, and blocking gap junctions with octanol reverses BDNF-induced synchrony.

It is known that delay neurons are mostly excitatory glutamatergic neurons expressing vesicular glutamate transporter 2 (VGLUT2) and that tonic neurons are mostly GABA- or glycinergic inhibitory neurons ${ }^{31}$. We also know from the pioneering studies of Perl and others that tonic neurons mainly possess an islet morphology and that delay neurons possess a radial morphology ${ }^{32}$. While the physiological correlates of the BDNF-related $\left[\mathrm{Ca}^{2+}\right]_{i}$ fluctuations are unclear, we do not think they are being driven by delay or tonic-firing neurons in the SDH. Unlike our analysis of the $\left[\mathrm{Ca}^{2+}\right]_{\mathrm{i}}$ fluctuations where we describe the 'silent' neurons, we did not observe any unmasking effect(s) of BDNF on the sEPSCs in the delay or tonic neurons. All neurons sampled in the control (naïve) condition generated visually appreciable, large-amplitude sEPSCs. The main depressive effect of BDNF on the sEPSCs (refer also to Supplemental Fig. 4) mirrored the main effect of BDNF on comparable properties for the $\left[\mathrm{Ca}^{2+}\right]_{\mathrm{i}}$ fluctuations. A summary of each dataset (Fig. 6, table) shows BDNF reduced the amplitude and area under the curve parameters for the $\left[\mathrm{Ca}^{2+}\right]_{\mathrm{i}}$ fluctuations and large sEPSCs. More nuanced effects were observed for frequency (reciprocal of interevent interval) and activation kinetics: BDNF increased the frequency of the large sEPSCs but had little to no effect on the frequency of the $\left[\mathrm{Ca}^{2+}\right]_{\mathrm{i}}$ fluctuations; BDNF increased activation of the large sEPSCs in the tonic neurons and had little to no effect on the delay neurons or $\left[\mathrm{Ca}^{2+}\right]_{\mathrm{i}}$ fluctuations. However, these data collectively suggest chronic BDNF may have reduced excitability in the delay and tonic SDH neurons, and this may provide a physiological basis for the depressive effect of chronic BDNF on the $\left[\mathrm{Ca}^{2+}\right]_{\mathrm{i}}$ fluctuations. On the contrary, the 'silent' neurons are predicted to have responded to chronic BDNF with $\left[\mathrm{Ca}^{2+}\right]_{\mathrm{i}}$ fluctuations of greater amplitude, total area, frequency, and presumably activation. Our working model (Fig. 6, bottom) posits that under naïve conditions many delay and tonic SDH neurons in organotypic spinal cord cultures are generally 


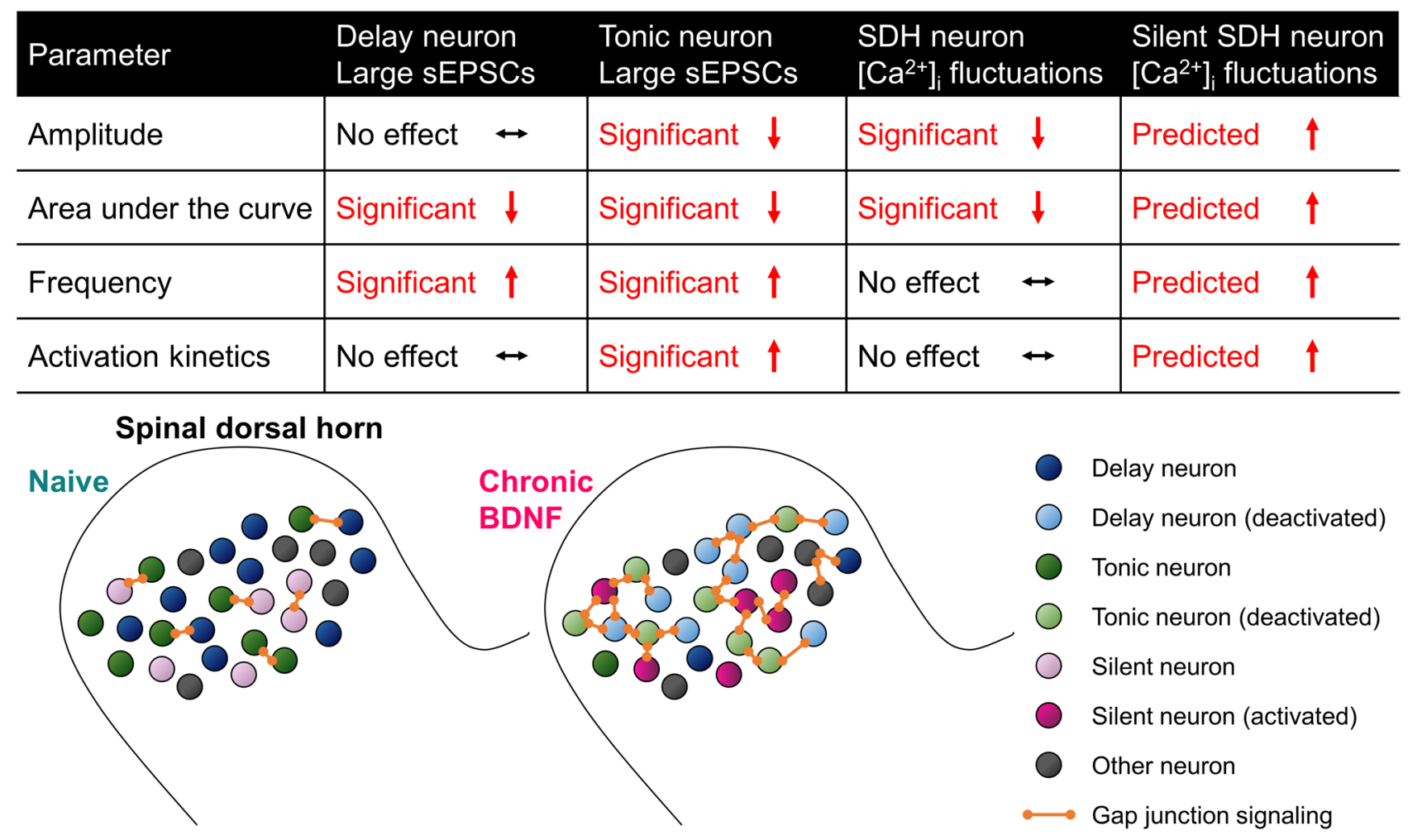

Figure 6. Working model summarizing the effects of chronic BDNF on SDH neurons in this study.

in an active state, with some gap junction coupling with neighboring neurons. Long-term exposure to BDNF causes a homeostatic shift that pushes the SDH into a dampened state marked by synchronous, low-level activity spread across the network via increased gap junction coupling. This synchronous activity in turn mitigates the BDNF-activated 'silent' neurons. From our pharmacology experiments (Fig. 4 and Supplementary Fig. 1), we also suggest that activity of GABA- and glycinergic inhibitory receptor systems are necessary to support BDNFinduced $\mathrm{Ca}^{2+}$ fluctuations. Given the role of GABA and glycine in sensory processing associated with chronic pain $^{33}$, it is possible that BDNF-induced fluctuations are important for pathophysiology of neuropathic pain.

Several types of oscillatory and/or rhythmic bursting activity have been previously observed in spinal cord organotypic cultures ${ }^{34,35}$ and in ex vivo slice preparations ${ }^{26,36-38}$. BDNF has also been shown to induce long term potentiation (LTP) in the spinal dorsal horn ${ }^{39}$ and is critically involved in mechanisms of synaptic plasticity, which may induce synchronous activity in different pain states. Synchronous activation of groups of spinal nociceptive neurons might contribute to the 'electric shock'-like sensations experienced by some neuropathic pain patients ${ }^{40}$. We hypothesize that $\left[\mathrm{Ca}^{2+}\right]_{\mathrm{i}}$ fluctuations leading to synchronous network activity may be attributed to the "shooting pains" that chronic pain patients may experience. In particular, "shooting pain" is very commonly experienced by patients with radicular lower back pain (sciatica) or trigeminal neuralgia. In these patients, stimulus movement elicits so-called "traveling waves" in which neural activity sweeps across the bodypart representation in somatosensory maps ${ }^{41}$. The mechanisms of this have been attributed in part to $\mathrm{Ca}^{2+}$ waves and gap junctions ${ }^{41,42}$. Therefore, given the properties of the $\left[\mathrm{Ca}^{2+}\right]_{\mathrm{i}}$ fluctuations we have identified, they would make a good candidate for the neural substrate of shooting pain. In a cohort of chemotherapy-induced peripheral neuropathy (CIPN) patients, some of whom reported shooting or burning pain in the hands or feet, serum nerve growth factor (NGF) levels were much higher compared with patients with painless or absent $\mathrm{CIPN}^{43}$. Therefore, there is a precedent for the correlation of neurotrophic factors related to BDNF to the incidence of shooting pain. Indeed it has been shown that NGF regulates the expression of $\mathrm{BDNF}^{44}$. It is has also been demonstrated that BDNF overexpression induces spasticity in rodent models of spinal cord injury, which may also explain the effect of BDNF on influencing network excitability in the dorsal horn ${ }^{45}$. With regards to the involvement of the dorsal horn in shooting pain, it has been shown that dorsal root entry zone lesioning (DREZotomy) successfully reduces shooting pain caused by brachial plexus root avulsion (BPRA) ${ }^{46}$. Also, in one patient with shooting pain caused by osteoid osteoma, a partial laminectomy was able to significantly relieve pain symptoms ${ }^{47}$. Ideally however, more experiments using human ex vivo tissue are needed to determine whether these particular $\left[\mathrm{Ca}^{2+}\right]_{\mathrm{i}}$ fluctuations correlate with shooting pain in patients.

\section{Materials and methods}

Ethics. All experimental protocols were approved by the University of Alberta Animal Care and Use Committee, which is charged with maintaining standards set forth by the Canadian Council on Animal Care. 
Defined-medium organotypic cultures of spinal cord slices. All procedures complied with the guidelines of the Canadian Council for Animal Care and the University of Alberta Health Sciences Laboratory Animal Services Welfare Committee. Spinal cords were isolated from embryonic (E13-14) rats and transverse slices $(300 \pm 25 \mu \mathrm{m})$ were cultured using the roller-tube technique ${ }^{20,22}$. Since tissue is obtained from embryos, sex could not be determined. Serum-free conditions were established after 5 days in vitro. Medium was exchanged with freshly prepared medium every 3-4 days. Slices were treated after 15-21 days in vitro for a period of 5-6 days with $50-200 \mathrm{ng} / \mathrm{ml}$ in serum-free medium as described previously ${ }^{20}$. Age-matched, untreated DMOTC slices served as controls.

Calcium imaging. Each organotypic slice was incubated for $1 \mathrm{~h}$ prior to imaging with the fluorescent $\mathrm{Ca}^{2+}$-indicator dye Fluo-4-AM (Molecular Probes, Invitrogen, Carlsbad, CA, USA). The conditions for incubating the dye were standardized across different slices to avoid uneven dye loading. After dye loading, the slice was transferred to a recording chamber and superfused with external solution containing (in $\mathrm{mM}$ ): $131 \mathrm{NaCl}, 2.5$ $\mathrm{KCl}, 1.2 \mathrm{NaH}_{2} \mathrm{PO}_{4}, 1.3 \mathrm{MgSO}_{4}, 26 \mathrm{NaHCO}_{3}, 25 \mathrm{D}$-glucose, and $2.5 \mathrm{CaCl}_{2}\left(20{ }^{\circ} \mathrm{C}\right.$, flow rate $\left.4 \mathrm{ml} / \mathrm{min}\right)$. Regions of interest (ROI) corresponding to individual cell bodies of neurons were identified based on morphology and size. Changes in $\mathrm{Ca}^{2+}$-fluorescence intensity evoked by a high $\mathrm{K}^{+}$solution $(20,35$, or $50 \mathrm{mM}, 90 \mathrm{~s}$ application) or other pharmacological agents, were measured in dorsal horn neurons with a confocal microscope equipped with an argon $(488 \mathrm{~nm})$ laser and filters (20 X XLUMPlanF1-NA-0.95 objective; Olympus FV300, Markham, Ontario, Canada). Full frame images $(512 \times 512$ pixels $)$ in a fixed xy plane were acquired at a scanning time of $0.8-1.08 \mathrm{~s} /$ frame $^{48}$. In some experiments, images were cropped to accommodate faster scan rates. Selected regions of interest were drawn around distinct cell bodies depicting neurons based on morphology as described previously ${ }^{49}$ and fluorescence intensity traces were generated with FluoView v.4.3 (Olympus).

Electrophysiology. Whole cell patch-clamp recordings were obtained from neurons in organotypic slice cultures under infrared differential interference contrast optics. Neurons selected for recording were located $250-800 \mu \mathrm{m}$ from the dorsal edge of the cultures in an area presumed to reflect the substantia gelatinosa and up to a depth of $100 \mu \mathrm{m}$ from the surface. Neurons were categorized according to their firing pattern in response to depolarizing current commands as tonic, delay, phasic, irregular, or transient ${ }^{30}$. Recordings were obtained with an NPI SEC-05LX amplifier (ALA Scientific Instruments, Westbury, NY, USA) in bridge balance or in discontinuous, single electrode, current or voltage-clamp mode. Neurons were sampled at $2 \mathrm{k} \mathrm{Hz}$ for $180 \mathrm{~s}$ when measuring sEPSCs. For recording, slices were superfused at room temperature $\left(\sim 22{ }^{\circ} \mathrm{C}\right)$ with $95 \% \mathrm{O} 2-5 \%$ $\mathrm{CO}_{2}$-saturated aCSF that contained (in mM) $127 \mathrm{NaCl}, 2.5 \mathrm{KCl}, 1.2 \mathrm{NaH}_{2} \mathrm{PO}_{4}, 26 \mathrm{NaHCO}_{3}, 1.3 \mathrm{MgSO}_{4}, 2.5$ $\mathrm{CaCl}_{2}$, and $25 \mathrm{D}$-glucose, $\mathrm{pH}$ 7.4. Patch pipettes were pulled from thin-walled borosilicate glass $(1.5 / 1.12 \mathrm{~mm}$ $\mathrm{OD} / \mathrm{ID}$; WPI, Sarasota, FL) to 5- to $10-\mathrm{M} \Omega$ resistances when filled with an internal solution containing (in $\mathrm{mM}$ ) 130 potassium gluconate, $1 \mathrm{MgCl} 2,2 \mathrm{CaCl} 2,10 \mathrm{HEPES}, 10$ EGTA, $4 \mathrm{Mg}$-ATP, and 0.3 Na-GTP, pH 7.2, 290-300 mosM.

Drugs and chemicals. Unless otherwise stated, all chemicals were from Sigma (St. Louis, MO, USA). Fluo-4 AM dye was dissolved in a mixture of dimethyl sulfoxide (DMSO) and 20\% pluronic acid (Invitrogen, Burlington, Ontario, Canada) to a $0.5 \mathrm{mM}$ stock solution and kept frozen until used. The dye was thawed and sonicated thoroughly before incubating with a DMOTC slice. TTX was dissolved in distilled water as a $1 \mathrm{mM}$ stock solution and stored at $-20^{\circ} \mathrm{C}$ until use. TTX was diluted to a final desired concentration of $1 \mu \mathrm{M}$ in external recording solution on the day of the experiment. Strychnine was prepared in a similar manner to TTX, and bicuculline (Tocris, Ballwin, MO, USA) was dissolved in DMSO as a $10 \mathrm{mM}$ stock solution. 6-cyano-7-nitroquinoxaline-2,3-dione (CNQX, Tocris), 2,3-dihydroxy-6-nitro-7-sulfamoyl-benzo[f]quinoxaline-2,3-dione (NBQX, Tocris) and N,N,H,-Trimethyl-5-[(tricyclo[3.3.1.13,7]dec-1-ylmethyl)amino]-1-pentanaminiumbromide hydrobromide (IEM-1460, Tocris) were prepared as $10 \mathrm{mM}$ stocks dissolved in distilled water, and $\mathrm{D}(-$ )-2-Amino-5-phosphonopentanoic acid (D-AP5, Tocris) was prepared as $50 \mathrm{mM}$ stocks dissolved in $30 \% 1 \mathrm{M}$ $\mathrm{NaOH}$. Riluzole was prepared as a $10 \mathrm{mM}$ stock, kyneurinic acid and GABA as $1 \mathrm{M}$ stocks, and nitrendipine as a $1 \mathrm{mM}$ stock made up in distilled water. These drugs were used at a 1:1000 dilution prepared freshly with external recording solution immediately prior to the start of experiments.

Data analysis and statistical testing. Raw time-lapse calcium fluorescence intensity and whole-cell voltage-clamp recordings were analyzed using the Frequency-Independent Biological Signal Identification (FIBSI) program ${ }^{24}$ written using the Anaconda v2019.7.0.0 (Anaconda, Inc, Austin, TX) distribution of Python v3.5.2 and the NumPy and matplotlib.pyplot libraries. The FIBSI program incorporates the Ramer-DouglasPeucker algorithm to detect significant waveforms against a time-dependent generated reference line using the least number of total points to represent said waveform, and provides quantitative measurements for each detected waveform. The calcium fluorescence traces were fit using a sliding median with a window size between 5 and $25 \mathrm{~s}$, and peaks below the sliding median line were traced together to form the reference line. The voltageclamp traces were fit using a sliding median with a window of $50 \mathrm{~ms}$, and peaks above the sliding median were traced together to form the reference line. A custom Python script was used to match sEPSCs detected in filtered voltage-clamp recordings with the unfiltered recordings. The detected sEPSCs for each neuron were clustered based on their amplitude, charge transfer, and interevent interval using the partitioning around medoids function (default settings, manhattan distance used) in the cluster v2.1.0 package in R v4.0.2 (2020-06-22; R Core Team, The R Foundation for Statistical Computing, Vienna, Austria). Each neuron was clustered independently. The average silhouette width method was used to select the optimal number of clusters for each neuron. Clusters were graphed using the plotly v4.9.2.1 package with dependency on ggplot2. The FIBSI source code, custom 
Python matching script, and a tutorial for using FIBSI are available on a GitHub repository (https://github.com/ rmcassidy/FIBSI_program).

Statistical analysis of the calcium fluctuations and sEPSCs were performed using Prism v8.2.1 (GraphPad Software, Inc, La Jolla, CA). The comparison between the proportion of 'silent' neurons in the naïve and BDNF treatment conditions was made using Fisher's exact test. The Brown-Forsythe and Welch ANOVA tests and Games-Howell post-hoc test (for $n>50$ ) were used to compare the effects of BDNF when the calcium fluctuations from all neurons were grouped together. To control for differences in recording times, the means for each fluctuation parameter (amplitude, AUC, etc.) were calculated for each neuron (fluctuations $<10 \%$ the maximal amplitude in each neuron were omitted to remove the effects of low-amplitude noise). The amplitude and AUC means were normalized across all neurons to permit direct comparisons. Normality was assessed using the D'Agostino \& Pearson omnibus test, and nonparametric comparisons between group medians were made using the Kruskal-Wallis test and Dunn's post-hoc test. Two-way repeated measures ANOVAs and Sidak's post-hoc tests were used to compare the pre- and post-treatment effects of octanol on the calcium fluctuations. Adjacency matrixes for neurons imaged together from the same organotypic culture were constructed using Pearson correlation matrixes; the FIBSI-processed calcium fluctuation recordings were used as input (each recording was normalized on a 0 to 1 scale). Next, the mean Pearson $r$ coefficient for each neuron within a culture was calculated using the Fisher $\mathrm{Z}$ transformation (steps shown in Fig. 3A). The mean Pearson $r$ coefficients in the naïve and BDNF conditions were not normally distributed, so the comparison between the two was made using a Mann-Whitney test. Comparisons between the pre- and post-treatment effects of octanol were first made using a Wilcoxon matched-pairs signed rank test, and then using a two-way repeated measures ANOVA and Sidak's post-hoc test. Paired t-tests were used for pharmacology experiments. Brown-Forsythe and Welch ANOVA tests and Games-Howell post-hoc test were used to compare the effects of BDNF on the sEPSC clusters. Statistical significant was set to $P<0.05$ and all reported $P$ values are two-tailed. Details for the statistical analyses can be found in Table 1.

Received: 9 November 2020; Accepted: 4 January 2021

Published online: 26 January 2021

\section{References}

1. Finnerup, N. B. et al. Neuropathic pain: an updated grading system for research and clinical practice. Pain 157, 1599-1606 (2016).

2. Alles, S. R. A. \& Smith, P. A. Etiology and pharmacology of neuropathic pain. Pharmacol. Rev. 70, 315-347 (2018).

3. Sandkuhler, J. Models and mechanisms of hyperalgesia and allodynia. Physiol. Rev. 89, 707-758 (2009).

4. Stemkowski, P. L. \& Smith, P. A. Sensory neurons, ion channels, inflammation and the onset of neuropathic pain. Can. J. Neurol. Sci. J. Can. Sci. Neurol. 39, 416-435 (2012).

5. Abdulla, F. A. \& Smith, P. A. Axotomy- and autotomy-induced changes in the excitability of rat dorsal root ganglion neurons. J. Neurophysiol. 85, 630-643 (2001).

6. Amir, R., Michaelis, M. \& Devor, M. Membrane potential oscillations in dorsal root ganglion neurons: Role in normal electrogenesis and neuropathic pain. J. Neurosci. Off. J. Soc. Neurosci. 19, 8589-8596 (1999).

7. Djouhri, L., Smith, T., Ahmeda, A., Alotaibi, M. \& Weng, X. Hyperpolarization-activated cyclic nucleotide-gated channels contribute to spontaneous activity in $\mathrm{L} 4 \mathrm{C}$-fiber nociceptors, but not $\mathrm{A} \beta$-non-nociceptors, after axotomy of $\mathrm{L} 5$-spinal nerve in the rat in vivo. Pain 159, 1392-1402 (2018).

8. Djouhri, L., Zeidan, A., Abd El-Aleem, S. A. \& Smith, T. Cutaneous A $\beta$-non-nociceptive, but not C-nociceptive, dorsal root ganglion neurons exhibit spontaneous activity in the streptozotocin rat model of painful diabetic neuropathy in vivo. Front. Neurosci. 14, 1-10 (2020).

9. Boakye, P. A. et al. Receptor dependence of BDNF actions in superficial dorsal horn: Relation to central sensitization and actions of macrophage colony stimulating factor 1. J. Neurophysiol. 121, 2308-2322 (2019).

10. Coull, J. A. et al. BDNF from microglia causes the shift in neuronal anion gradient underlying neuropathic pain. Nature 438, 1017-1021 (2005).

11. Guan, Z. et al. Injured sensory neuron-derived CSF1 induces microglial proliferation and DAP12-dependent pain. Nat. Neurosci. 19, 94-101 (2016).

12. Scholz, J. \& Woolf, C. J. The neuropathic pain triad: Neurons, immune cells and glia. Nat. Neurosci. 10, 1361-1368 (2007).

13. Tsuda, M. et al. P2X4 receptors induced in spinal microglia gate tactile allodynia after nerve injury. Nature 424, 778-783 (2003).

14. Ulmann, L. et al. Up-regulation of P2X4 receptors in spinal microglia after peripheral nerve injury mediates BDNF release and neuropathic pain. J. Neurosci. 28, 11263-11268 (2008).

15. Woolf, C. J. Evidence for a central component of post-injury pain hypersensitivity. Nature 306, 686-688 (1983).

16. Coull, J. A. et al. Trans-synaptic shift in anion gradient in spinal lamina I neurons as a mechanism of neuropathic pain. Nature 424, 938-942 (2003).

17. Ferrini, F. et al. Differential chloride homeostasis in the spinal dorsal horn locally shapes synaptic metaplasticity and modalityspecific sensitization. Nat. Commun. 11, 3935 (2020).

18. $\mathrm{Lu}, \mathrm{V}$. B. et al. Brain-derived neurotrophic factor drives the changes in excitatory synaptic transmission in the rat superficial dorsal horn that follow sciatic nerve injury. J. Physiol. 587, 1013-1032 (2009).

19. Biggs, J. E., Lu, V. B., Stebbing, M. J., Balasubramanyan, S. \& Smith, P. A. Is BDNF sufficient for information transfer between microglia and dorsal horn neurons during the onset of central sensitization?. Mol. Pain 6, 44 (2010).

20. Lu, V. B., Ballanyi, K., Colmers, W. F. \& Smith, P. A. Neuron type-specific effects of brain-derived neurotrophic factor in rat superficial dorsal horn and their relevance to 'central sensitization'. J. Physiol. 584, 543-563 (2007).

21. Lu, V. B. et al. Substantia Gelatinosa neurons in defined-medium organotypic slice culture are similar to those in acute slices from young adult rats. Pain 121, 261-275 (2006).

22. Biggs, J. E. et al. Defined medium organotypic cultures of spinal cord put 'pain in a dish' \#. Trans. Isolat. Cent. Nerv. Syst. Circuits 73, 405-436 (2012).

23. Boakye, P. A. et al. Characterization of Superficial Dorsal Horn Neurons from "Tamamaki" Mice and Stability of their GAD67-EGFP Phenotype in Defined-Medium Organotypic Culture. (2018).

24. Cassidy, R. M. et al. Frequency-independent biological signal identification (FIBSI): A free program that simplifies intensive analysis of non-stationary time series data. bioRxiv https://doi.org/10.1101/2020.05.29.123042 (2020).

25. Smith, P. A. BDNF: No gain without pain?. Neuroscience 283, 107-123 (2014). 
26. Asghar, A. U. R. et al. Oscillatory activity within rat substantia gelatinosa in vitro: A role for chemical and electrical neurotransmission. J. Physiol. 562, 183-198 (2005).

27. Chapman, R. J., La Corte, P. F. C., Asghar, A. U. R. \& King, A. E. Network-based activity induced by 4-aminopyridine in rat dorsal horn in vitro is mediated by both chemical and electrical synapses. J. Physiol. 587, 2499-2510 (2009).

28. Ochalski, P. A. Y., Frankenstein, U. N., Hertzberg, E. L. \& Nagy, J. I. Connexin-43 in rat spinal cord: Localization in astrocytes and identification of heterotypic astro-oligodendrocytic gap junctions. Neuroscience 76, 931-945 (1996).

29. Kay, C. W. P., Ursu, D., Sher, E. \& King, A. E. The role of Cx36 and Cx43 in 4-aminopyridine-induced rhythmic activity in the spinal nociceptive dorsal horn: an electrophysiological study in vitro. Physiol. Rep. 4, 1-10 (2016).

30. Lu, V. B., Colmers, W. F. \& Smith, P. A. Long-term actions of BDNF on inhibitory synaptic transmission in identified neurons of the rat substantia gelatinosa. J. Neurophysiol. 108, 441-452 (2012).

31. Yasaka, T., Tiong, S. Y., Hughes, D. I., Riddell, J. S. \& Todd, A. J. Populations of inhibitory and excitatory interneurons in lamina II of the adult rat spinal dorsal horn revealed by a combined electrophysiological and anatomical approach. Pain 151, 475-488 (2010).

32. Grudt, T. J. \& Perl, E. R. Correlations between neuronal morphology and electrophysiological features in the rodent superficial dorsal horn. J. Physiol. 540, 189-207 (2002).

33. Hughes, D. I. \& Todd, A. J. Central nervous system targets: inhibitory interneurons in the spinal cord. Neurother. J. Am. Soc. Exp. Neurother. 17, 874-885 (2020).

34. Fabbro, A., Pastore, B., Nistri, A. \& Ballerini, L. Activity-independent intracellular Ca2+ oscillations are spontaneously generated by ventral spinal neurons during development in vitro. Cell Calcium 41, 317-329 (2007).

35. Sibilla, S. et al. The patterns of spontaneous $\mathrm{Ca} 2+$ signals generated by ventral spinal neurons in vitro show time-dependent refinement. Eur. J. Neurosci. 29, 1543-1559 (2009).

36. Bardoni, R. et al. Glutamate-mediated astrocyte-to-neuron signalling in the rat dorsal horn. J. Physiol. 588, 831-846 (2010).

37. Li, J. \& Baccei, M. L. Pacemaker neurons within newborn spinal pain circuits. J. Neurosci. 31, 9010-9022 (2011).

38. Ruscheweyh, R. \& Sandkühler, J. Long-range oscillatory Ca2+ waves in rat spinal dorsal horn. Eur. J. Neurosci. 22, 1967-1976 (2005).

39. Zhou, L.-J. et al. Microglia are indispensable for synaptic plasticity in the spinal dorsal horn and chronic pain. Cell Rep. 27, 38443859.e6 (2019).

40. Baron, R., Binder, A. \& Wasner, G. Neuropathic pain: Diagnosis, pathophysiological mechanisms, and treatment. Lancet Neurol. 9, 807-819 (2010).

41. Defrin, R., Brill, S., Goor-Arieh, I., Wood, I. \& Devor, M. 'Shooting pain' in lumbar radiculopathy and trigeminal neuralgia, and ideas concerning its neural substrates. Pain 161, 308-318 (2020).

42. Hanani, M. Intercellular communication in sensory ganglia by purinergic receptors and gap junctions: Implications for chronic pain. Brain Res. 1487, 183-191 (2012).

43. Velasco, R. et al. Neuropathic pain and nerve growth factor in chemotherapy-induced peripheral neuropathy: Prospective clinicalpathological study. J. Pain Symptom Manage. 54, 815-825 (2017).

44. Pezet, S. \& McMahon, S. B. Neurotrophins: mediators and modulators of pain. Annu. Rev. Neurosci. 29, 507-538 (2006).

45. Fouad, K., Bennett, D. J., Vavrek, R. \& Blesch, A. Long-term viral brain-derived neurotrophic factor delivery promotes spasticity in rats with a cervical spinal cord hemisection. Front. Neurol. 4, 00187 (2013).

46. Yanagisawa, T. et al. MEG-BMI to control phantom limb pain. Neurol. Med. Chir. 58, 327-333 (2018).

47. Sarmiento, J. M., Chan, J. L., Cohen, J. D., Mukherjee, D. \& Chu, R. M. L5 osteoid osteoma treated with partial laminectomy and cement augmentation. Cureus 11, e4239 (2019).

48. Ruangkittisakul, A. et al. High sensitivity to neuromodulator-activated signaling pathways at physiological [K+] of confocally imaged respiratory center neurons in on-line-calibrated newborn rat brainstem slices. J. Neurosci. Off. J. Soc. Neurosci. 26, 1187011880 (2006).

49. Biggs, J. E. et al. Analysis of the long-term actions of gabapentin and pregabalin in dorsal root ganglia and substantia gelatinosa. J. Neurophysiol. https://doi.org/10.1152/jn.00168.2014 (2014).

\section{Acknowledgements}

We thank Edgar Walters for useful discussions. We thank Klaus Ballanyi and Araya Ruangkittisakul for use of and technical assistance with confocal imaging equipment.

We acknowledge funding from the Canadian Institutes for Health Research. We acknowledge funding from the research endowment fund of the Department of Anesthesiology and Critical Care Medicine, University of New Mexico Health Sciences Center.

\section{Author contributions}

S.R.A.A. performed $\mathrm{Ca}^{2+}$ imaging experiments, data analysis and wrote first draft of the manuscript. M.A.O. performed FIBSI analysis, constructed figures, wrote and edited the manuscript. P.A.S. conceptualized experiments, supervised experiments and edited the manuscript. V.B.L. performed $\mathrm{Ca}^{2+}$ imaging and electrophysiology experiments, data analysis and edited the manuscript. R.M.C. assisted with FIBSI analysis.

\section{Competing interests}

The authors declare no competing interests.

\section{Additional information}

Supplementary Information The online version contains supplementary material available at https://doi. org/10.1038/s41598-021-81269-6.

Correspondence and requests for materials should be addressed to S.R.A.A.

Reprints and permissions information is available at www.nature.com/reprints.

Publisher's note Springer Nature remains neutral with regard to jurisdictional claims in published maps and institutional affiliations. 
(c) (i) Open Access This article is licensed under a Creative Commons Attribution 4.0 International cc) License, which permits use, sharing, adaptation, distribution and reproduction in any medium or format, as long as you give appropriate credit to the original author(s) and the source, provide a link to the Creative Commons licence, and indicate if changes were made. The images or other third party material in this article are included in the article's Creative Commons licence, unless indicated otherwise in a credit line to the material. If material is not included in the article's Creative Commons licence and your intended use is not permitted by statutory regulation or exceeds the permitted use, you will need to obtain permission directly from the copyright holder. To view a copy of this licence, visit http://creativecommons.org/licenses/by/4.0/.

(C) The Author(s) 2021 